\title{
EFICIÊNCIA DOS HERBICIDAS OXADIAZIL, OXADIAZON, OXYFLUORFEN E IMAZAPYR SOBRE A CULTURA DE PINUS ${ }^{1}$
}

\author{
WILSON DA SILVA ${ }^{2}$, FRANCISCO A. FERREIRA ${ }^{3}$, JOSÉ F. DA SILVA ${ }^{3}$, LUÍS E. FIRMINO ${ }^{4}$
}

\section{RESUMO}

Avaliou-se a seletividade de alguns herbicidas para mudas de Pinus caribaea var. Hondurensis e suas eficiências no controle de plantas daninhas. As mudas com $20 \mathrm{~cm}$ de altura, produzidas em tubetes, foram transplantadas num espaçamento de $2,0 \mathrm{~m} \times 2,0 \mathrm{~m}$, em parcelas de quatro fileiras com $10,0 \mathrm{~m}$ de comprimento. Os tratamentos utilizados foram oxadiazil $(600 ; 800 \mathrm{e}$ $\left.1000 \mathrm{~g} \mathrm{ha}^{-1}\right)$, oxadiazon $\left(1140 ; 1520\right.$ e $\left.1900 \mathrm{~g} \mathrm{ha}^{-1}\right)$, oxyfluorfen (720 $\left.\mathrm{g} \mathrm{ha}^{-1}\right)$, imazapyr $\left(250 \mathrm{~g} \mathrm{ha}^{-1}\right) \mathrm{e}$ testemunhas (capinada e sem capina), dispostos em blocos ao acaso, com quatro repetições. Os herbicidas foram aplicados sete dias após o transplante, usando-se pulverizador costal, pressurizado com $\mathrm{CO}_{2}$, calibrado para $200 \mathrm{~L} \mathrm{ha}^{-1}$ de calda. As principais plantas daninhas com maior infestação foram: Brachiaria plantaginea e
Ipomoea grandifolia e com menor infestação: Galinsoga parviflora e Bidens pilosa. O oxadiazil apresentou excelente controle de B. plantaginea, $I$. grandifolia e G. parviflora, não sendo eficiente para controle de B. pilosa, embora proporcionasse controle superior ao observado pelo oxadiazon. $\mathrm{O}$ oxadiazon foi eficiente até 45 dias após o tratamento para B. plantaginea, G. parviflora e até 90 dias para I. grandifolia. Tanto o oxyfluorfen quanto o imazapyr apresentaram excelente controle das plantas daninhas. De todos os herbicidas avaliados, apenas o imazapyr não deve ser recomendado para aplicação sobre o dossel de Pinus caribaea var. Hondurensis, em face da toxicidade provocada.

Palavras chave: Pinus caribaea var. Hondurensis, herbicidas, planta daninha, controle.

\section{ABSTRACT}

\section{Efficiency of the oxadiazil, oxadiazon, oxyfluorfen and imazapyr herbicides on Pinus cropping}

The selectivity of some herbicides were evaluated on Pinus caribaea var. Hondurensis seedlings as well as these herbicide efficiencies in the weed control. When the tubule-produced seedlings were $20 \mathrm{~cm}$ height they were transplanted at 2,0 $\mathrm{m} \times 2,0 \mathrm{~m}$ spacings in fourrows plots with $10,0 \mathrm{~m}$ length. The treatments with oxadiazil $\left(600 ; 800\right.$ and $\left.1000 \mathrm{~g} \mathrm{ha}^{-1}\right)$, oxadiazon $\left(1140 ; 1520\right.$ and $\left.1900 \mathrm{~g} \mathrm{ha}^{-1}\right)$, oxyfluorfen (720 $\left.\mathrm{g} \mathrm{ha}^{-1}\right)$, imazpyr $\left(250 \mathrm{~g} \mathrm{ha}^{-1}\right)$ and controls (weeded and without weeding) were disposed in a randomized block design with four replications. Seven days after transplantation, the herbicides were applied using a $\mathrm{CO}_{2}$ pressured costal sprayer which was calibrated to provide a $200 \mathrm{~L} \mathrm{ha}^{-1}$ solution. The main weeds causing a larger infestation were Brachiaria plantaginea and Ipomoea grandifolia while Galinsoga parviflora and Bidens pilosa were responsible for a minor one. The oxadiazil showed an excellent control on B. plantaginea, I. grandifolia and G. parviflora; although not being efficient to control

\footnotetext{
${ }^{1}$ Recebido para publicação em 12/08/98 e na forma revisada em 10/02/99.

${ }^{2}$ Doutor em Fitotecnia. Departamento de Fitotecnia - Universidade Federal de Viçosa - Campus Univ. CEP: 36.571.000.

${ }^{3}$ Prof. Titular. Departamento de Fitotecnia - Universidade Federal de Viçosa - Campus Univ. CEP: 36.571.000.

${ }^{4}$ Pós-graduando em Fitotecnia. Departamento de Fitotecnia - Universidade Federal de Viçosa - Campus Univ. CEP: 36.571.000.
} 
Bidens pilosa, it provided a superior control to the one observed in oxadiazon. Oxadiazon showed efficiency until 45 days after the treatment for $B$. plantaginea, G. parviflora and until 90 days for $I$. grandifolia as well. Either oxyfluorfen or imazapyr showed an excellent weed control.

\section{INTRODUÇÃO}

A cultura do Pinus apresenta crescimento inicial lento, necessitando de cuidados especiais quanto ao controle de plantas daninhas, por um período maior que a cultura do Eucalyptus.

A composição específica da comunidade infestante é fator de fundamental importância na determinação do grau de interferência, pois as espécies integrantes dessa comunidade variam bastante em relação aos seus hábitos de crescimento e exigências em recursos do meio. As espécies perenes dos gêneros Panicum $e$ Brachiaria (Pitelli \& Karan, 1988), assim como as espécies Imperata brasiliensis e Pteridium aquilinum (Zen, 1987) e as anuais Brachiaria plantaginea, Bidens pilosa, Ipomoea spp, Agerantum conyzoides, Galinsoga parviflora (Silva, 1995), são bastante prejudiciais nas fases iniciais de crescimento das espécies florestais. Voeller et al. (1974) afirmam ser a umidade do solo o fator de competição mais importante entre as gramíneas e o Pinus poderosa, porque as raízes das gramíneas desenvolvem mais rápido e, por isso, exploram melhor a umidade do solo, principalmente sob condições de estresse hídrico. Além da competição por água, as mudas das espécies florestais que crescem sob interferência de plantas daninhas também podem apresentar deficiência de alguns nutrientes, como consequiência, também, de maior habilidade dessas espécies em absorver tais nutrientes (Davies, 1990).

As características da cultura e das plantas daninhas e o período de convivência, as condições edafoclimáticas e os tratos culturais exercem grande influência na competição (Blesdale, 1960 e Agundis et al., 1962 - 1963).
Among the herbicides evaluated, only imazapyr must not be recommended for "on the top" application for Pinus caribeae var. Hondurensis, due to the toxicity provoked.

Key words: Pinus caribeae var. Hondurensis, herbicides, weed, control.
Neste trabalho objetivou-se avaliar a seletividade e a eficiência de alguns herbicidas sobre o controle de plantas daninhas na implantação da cultura de $P$. caribaea var. Hondurensis.

\section{MATERIAL E MÉTODOS}

O ensaio foi conduzido, em condições de campo, na Estação Experimental de Coimbra, Coimbra - MG, pertencente ao Departamento de Fitotecnia da Universidade Federal de Viçosa MG, em um Podzólico Vermelho - Amarelo Câmbico, fase terraço, textura argilosa, constituído por $23 \%$ de areia, $17 \%$ de silte, $60 \%$ de argila e o teor de matéria orgânica de $2,2 \%$ e pH 5,2.

Os tratamentos avaliados foram oxadiazil (600, 800 e $\left.1000 \mathrm{~g} \mathrm{ha}^{-1}\right)$, oxadiazon $(1140,1520$, $\left.1900 \mathrm{~g} \mathrm{ha}^{-1}\right)$, oxyfluorfen $\left(720 \mathrm{~g} \mathrm{ha}^{-1}\right)$, imazapyr $\left(250 \mathrm{~g} \mathrm{ha}^{-1}\right)$, e as testemunhas (capinada e sem capina), dispostos em blocos ao acaso com quatro repetições.

As mudas com $20 \mathrm{~cm}$ de altura foram produzidas em tubetes, transplantadas para as parcelas experimentais constituídas de quatro fileiras de 10,0 $\mathrm{m}$ de comprimento, espaçadas de $2,0 \mathrm{~m}$ entre fileiras e $2,0 \mathrm{~m}$ entre plantas, perfazendo um total de 20 plantas por parcela.

Os herbicidas foram aplicados sobre as mudas de pinus, sete dias após o transplante, estando as plantas daninhas no início da germinação. Foi usado pulverizador costal pressurizado com $\mathrm{CO}_{2}$, dotado de uma barra com cinco bicos teejet leque 80.03 , espaçados de $50 \mathrm{~cm}$ entre si, com uma pressão de $30 \mathrm{lb}$ por $\mathrm{pl}^{2}$, aplicando $200 \mathrm{~L} \mathrm{ha}^{-1}$ de calda. O solo estava úmido, o céu estava claro, sem nuvens, e a velocidade do vento era próxima a zero. A 
temperatura do ar era de $27^{\circ} \mathrm{C}$ e a umidade relativa do ar $80 \%$.

Os efeitos dos herbicidas sobre as mudas de pinus foram avaliados aos 15 e 30 dias após o tratamento (DAT), de acordo com os sintomas de toxicidade numa escala de 0 (zero) a $100 \%$, em que 0 (zero) equivale a nenhum dano visível na planta e 100, à morte da planta (Frans, 1972).

A avaliação de controle das plantas daninhas foi feita aos 30, 45, 70 e 90 DAT, adotando a escala visual, de 0,0 (ausência de controle) a 100 (controle total). Nessas épocas de avaliações, as principais plantas infestantes eram: Bracharia plantaginea, Ipomoea grandifolia, Bidens pilosa e Galinsoga parviflora.

Os dados do controle das plantas daninhas, para as quatro épocas, em cada espécie, foram submetidos à Análise de Variância e os tratamentos foram comparados pelo teste Tukey, a $5 \%$ de probabilidade.

\section{RESULTADOS E DISCUSSÃO}

As espécies predominantes foram Ipomoea grandifolia e Brachiaria plantaginea. Bidens pilosa e Galinsoga parviflora ocorreram com menor intensidade e mais desuniformidade nas parcelas e testemunhas.

Em razão de o pinus possuir um crescimento inicial lento e a sua arquitetura foliar não ser suficiente para boa cobertura do solo até 90 dias, a cultura mostra-se muito sensível à competição, com diversas espécies infestantes.

O oxadiazil, em doses superior a $60 \mathrm{~g} \mathrm{ha}^{-1}$, proporcionou eficiente controle Ipomoea grandifolia, acima de 95\%, até aos 90 DAT (Tabela 1). O oxyfluorfen na dose $720 \mathrm{~g} \mathrm{ha}^{-1}$ apresentou resultados semelhantes ao oxadiazil, apresentando 92,5\% de controle, aos 90 DAT. Para essa mesma espécie, oxadiazon propiciou um controle acima de 97\%, aos 45 (dias após o tratamento), nas três doses testadas. O imazapyr na dose $250 \mathrm{~g} \mathrm{ha}^{-1}$ apresentou $100 \%$ de controle, até 90 DAT.

TABELA 1.- Percentagem de controle de Ipomoea grandifolia aos 30, 45, 70 e 90 dias, após a aplicação dos herbicidas, em Pinus caribea var. Hondurensis.

\begin{tabular}{lcrrrr}
\hline \multicolumn{1}{c}{ Tratamentos } & $\begin{array}{c}\text { Doses } \\
\left(\mathrm{g} \mathrm{ha}^{-1}\right)\end{array}$ & 30 DAT & 45 DAT & 70 DAT & 90 DAT \\
\hline testemunha s/ capina & - & $0,0 \mathrm{c}$ & $0,0 \mathrm{c}$ & $0,0 \mathrm{e}$ & $0,0 \mathrm{~b}$ \\
testemunha c/ capina & - & $100,0 \mathrm{a}$ & $100,0 \mathrm{a}$ & $100,0 \mathrm{a}$ & $100,0 \mathrm{a}$ \\
oxadiazil & 600 & $100,0 \mathrm{a}$ & $100,0 \mathrm{a}$ & $96,8 \mathrm{bc}$ & $89,5 \mathrm{~b}$ \\
oxadiazil & 800 & $100,0 \mathrm{a}$ & $100,0 \mathrm{a}$ & $98,3 \mathrm{ab}$ & $95,8 \mathrm{ab}$ \\
oxadiazil & 1000 & $100,0 \mathrm{a}$ & $100,0 \mathrm{a}$ & $99,5 \mathrm{a}$ & $95,8 \mathrm{ab}$ \\
oxadiazon & 1140 & $97,0 \mathrm{~b}$ & $97,0 \mathrm{ab}$ & $91,3 \mathrm{~d}$ & $88,8 \mathrm{~b}$ \\
oxadiazon & 1520 & $100,0 \mathrm{a}$ & $100,0 \mathrm{a}$ & $95,5 \mathrm{c}$ & $94,5 \mathrm{ab}$ \\
oxadiazon & 1900 & $100,0 \mathrm{a}$ & $97,8 \mathrm{ab}$ & $95,8 \mathrm{c}$ & $92,0 \mathrm{~b}$ \\
oxyfluorfen & 720 & $100,0 \mathrm{a}$ & $100,0 \mathrm{a}$ & $98,8 \mathrm{ab}$ & $92,5 \mathrm{~b}$ \\
imazapyr & 250 & $100,0 \mathrm{a}$ & $100,0 \mathrm{a}$ & $100,0 \mathrm{a}$ & $100,0 \mathrm{a}$ \\
\hline CV $(\%)$ & & 1,2 & 1,3 & 1,1 & 3,6 \\
\hline
\end{tabular}

Médias seguidas pela mesma letra, em uma coluna, não diferem entre si a 5\% de probabilidade, pelo teste de Tukey. 
O controle de Bidens pilosa pelo oxadiazil e oxadiazon, na dose intermediária e na maior dose, não ultrapassou 77 e $69,5 \%$, respectivamente, até aos 45 dias após tratamento (Tabela 2). O oxyfluorfen, até 45 (dias após tratamento), proporcionou $100 \%$ de controle, reduzindo-se para 87,5\%, aos 90 DAT. O imazapyr, aos 90 DAT, apresentou $100 \%$ de controle dessa espécie.

TABELA 2. Percentagem de controle de Bidens pilosa aos 30, 45, 70 e 90 dias após a aplicação dos herbicidas, em Pinus caribea var. Hondurensis.

\begin{tabular}{lcrrrr}
\hline \multicolumn{1}{c}{ Tratamentos } & Doses & \multicolumn{5}{c}{ Dias após as aplicações } \\
& $\left(\mathrm{g} \mathrm{ha}^{-1}\right)$ & 30 DAT & 45 DAT & $70 \mathrm{DAT}$ & $90 \mathrm{DAT}$ \\
\hline testemunha s/ capina & - & $0,0 \mathrm{f}$ & $0,0 \mathrm{f}$ & $0,0 \mathrm{e}$ & $0,0 \mathrm{e}$ \\
testemunha c/ capina & - & $100,0 \mathrm{a}$ & $100,0 \mathrm{a}$ & $100,0 \mathrm{a}$ & $100,0 \mathrm{a}$ \\
oxadiazil & 600 & $45,0 \mathrm{e}$ & $35,5 \mathrm{e}$ & $14,3 \mathrm{de}$ & $13,7 \mathrm{~d}$ \\
oxadiazil & 800 & $61,3 \mathrm{~d}$ & $59,5 \mathrm{~d}$ & $19,3 \mathrm{~d}$ & $16,3 \mathrm{~cd}$ \\
oxadiazil & 1000 & $78,3 \mathrm{~b}$ & $76,3 \mathrm{~b}$ & $45,5 \mathrm{~b}$ & $21,3 \mathrm{~cd}$ \\
oxadiazon & 1140 & $68,8 \mathrm{c}$ & $75,0 \mathrm{~cd}$ & $38,3 \mathrm{c}$ & $26,3 \mathrm{c}$ \\
oxadiazon & 1520 & $73,8 \mathrm{bc}$ & $69,5 \mathrm{bc}$ & $18,8 \mathrm{~d}$ & $13,8 \mathrm{~d}$ \\
oxadiazon & 1900 & $72,3 \mathrm{c}$ & $68,8 \mathrm{c}$ & $15,0 \mathrm{de}$ & $12,5 \mathrm{~d}$ \\
oxyfluorfen & 720 & $100,0 \mathrm{a}$ & $100,0 \mathrm{a}$ & $90,0 \mathrm{a}$ & $87,5 \mathrm{~b}$ \\
imazapyr & 250 & $100,0 \mathrm{a}$ & $100,0 \mathrm{a}$ & $100,0 \mathrm{a}$ & $100,0 \mathrm{a}$ \\
\hline CV $(\%)$ & 3,7 & 4,5 & 13,5 & 11,3 \\
\hline
\end{tabular}

Médias seguidas pela mesma letra, em uma coluna, não diferem entre si a 5\% de probabilidade, pelo teste de Tukey.

O oxadiazon e o oxadiazil foram pouco eficientes no controle de Bidens pilosa. Os demais herbicidas, oxyfluorfen e imazapyr foram altamente eficientes para o controle dessa espécie. Silva et al. (1995) encontraram resultados semelhantes com oxyfluorfen, até 180 dias após a aplicação.

Com relação à eficiência de controle da Brachiaria plantaginea, o oxadiazil nas doses de 800 e $1.000 \mathrm{~g} \mathrm{ha}^{-1}$, proporcionou um controle acima de 92,5\%, até aos 70 DAT, reduzindo-se, para $87,5 \%$, aos 90 DAT. Esse produto superou, em todas as doses, a eficiência do oxadiazon, que apresentou controle de apenas $72 \%$, até aos 45 DAT (Tabela 3). Oxyfluorfen apresentou controle de $94 \%$ de controle de B. plantaginea, até aos 70
DAT, reduzindo-se para $88 \%$, aos 90 DAT. O imazapyr apresentou $100 \%$ de controle dessa espécie, também para a mesma época de aplicação.

O controle de Galinsoga parviflora pelo oxadiazil foi de $92,5 \%$, até aos 45 DAT. Aos 90 dias após tratamento, o controle foi reduzido para 72,5 e $86,3 \%$ nas doses 600 e $1.000 \mathrm{~g} \mathrm{ha}^{-1}$, respectivamente. $\mathrm{O}$ controle dessa espécie com o oxadiazon foi menor que o do oxadiazil, não ultrapassando $90 \%$ na maior dose $\left(1.900 \mathrm{~g} \mathrm{ha}^{-1}\right)$, aos 45 DAT. Após esse período, a eficiência de controle reduziu, significativamente, (Tabela 4). Tanto o oxyfuorfen quanto ao imazapyr apresentaram excelente controle de Galinsoga parviflora, até aos 90 DAT. 
TABELA 3. Percentagem de controle de Brachiaria plantaginea aos 30, 45, 70 e 90 dias após a aplicação dos herbicidas, em Pinus caribea var. Hondurensis.

\begin{tabular}{lcrrrrr}
\hline \multicolumn{1}{c}{ Tratamentos } & Doses & \multicolumn{5}{c}{ Dias após as aplicações } \\
& $\left(\mathrm{g} \mathrm{ha}^{-1}\right)$ & $30 \mathrm{DAT}$ & $45 \mathrm{DAT}$ & $70 \mathrm{DAT}$ & $90 \mathrm{DAT}$ \\
\hline testemunha s/ capina & - & $0,0 \mathrm{~d}$ & $0,0 \mathrm{~d}$ & $0,0 \mathrm{e}$ & $0,0 \mathrm{c}$ \\
testemunha c/ capina & - & $100,0 \mathrm{a}$ & $100,0 \mathrm{a}$ & $100,0 \mathrm{a}$ & $100,0 \mathrm{a}$ \\
oxadiazil & 600 & $87,5 \mathrm{~b}$ & $84,2 \mathrm{~b}$ & $88,3 \mathrm{ab}$ & $76,3 \mathrm{abc}$ \\
oxadiazil & 800 & $98,2 \mathrm{a}$ & $96,5 \mathrm{a}$ & $92,5 \mathrm{a}$ & $88,8 \mathrm{ab}$ \\
oxadiazil & 1000 & $100,0 \mathrm{a}$ & $98,0 \mathrm{a}$ & $94,8 \mathrm{a}$ & $87,5 \mathrm{ab}$ \\
oxadiazon & 1140 & $75,0 \mathrm{c}$ & $72,0 \mathrm{c}$ & $63,8 \mathrm{~d}$ & $50,0 \mathrm{~d}$ \\
oxadiazon & 1520 & $87,8 \mathrm{~b}$ & $82,8 \mathrm{~b}$ & $77,5 \mathrm{bc}$ & $66,3 \mathrm{bcd}$ \\
oxadiazon & 1900 & $83,8 \mathrm{~b}$ & $83,8 \mathrm{~b}$ & $68,5 \mathrm{~cd}$ & $52,5 \mathrm{~cd}$ \\
oxyfluorfen & 720 & $97,8 \mathrm{a}$ & $97,0 \mathrm{a}$ & $94,0 \mathrm{a}$ & $88,0 \mathrm{ab}$ \\
imazapyr & 250 & $100,0 \mathrm{a}$ & $100,0 \mathrm{a}$ & $100,0 \mathrm{a}$ & $100,0 \mathrm{a}$ \\
\hline CV $(\%)$ & 2,6 & 3,2 & 6,3 & 14,3 \\
\hline
\end{tabular}

Médias seguidas pela mesma letra, em uma coluna, não diferem entre si a 5\% de probabilidade, pelo teste de Tukey.

TABELA 4. Percentagem de controle de Galisoga Parviflora aos 30, 45, 70 e 90 dias após a aplicação dos herbicidas, em Pinus caribea var. hondurensis.

\begin{tabular}{lccrrrr}
\hline \multicolumn{1}{c}{ Tratamentos } & $\begin{array}{c}\text { Doses } \\
\left(\mathrm{g} \mathrm{ha}^{-1}\right)\end{array}$ & $30 \mathrm{DAT}$ & $45 \mathrm{DAT}$ & $70 \mathrm{DAT}$ & $90 \mathrm{DAT}$ \\
\hline testemunha s/ capina & - & $0,0 \mathrm{~d}$ & $0,0 \mathrm{~d}$ & $0,0 \mathrm{f}$ & $0,0 \mathrm{e}$ \\
testemunha c/ capina & - & $100,0 \mathrm{a}$ & $100,0 \mathrm{a}$ & $100,0 \mathrm{a}$ & $100,0 \mathrm{a}$ \\
oxadiazil & 600 & $92,3 \mathrm{abc}$ & $92,5 \mathrm{ab}$ & $80,0 \mathrm{c}$ & $72,5 \mathrm{bc}$ \\
oxadiazil & 800 & $95,8 \mathrm{ab}$ & $94,5 \mathrm{ab}$ & $89,3 \mathrm{~b}$ & $85,0 \mathrm{ab}$ \\
oxadiazil & 1000 & $97,8 \mathrm{ab}$ & $96,8 \mathrm{ab}$ & $91,0 \mathrm{~b}$ & $86,3 \mathrm{ab}$ \\
oxadiazon & 1140 & $87,0 \mathrm{~cd}$ & $83,3 \mathrm{c}$ & $51,5 \mathrm{e}$ & $46,3 \mathrm{~d}$ \\
oxadiazon & 1520 & $85,0 \mathrm{~d}$ & $83,8 \mathrm{c}$ & $57,5 \mathrm{de}$ & $53,8 \mathrm{~d}$ \\
oxadiazon & 1900 & $92,3 \mathrm{bcd}$ & $90,0 \mathrm{bc}$ & $62,5 \mathrm{~d}$ & $58,8 \mathrm{~cd}$ \\
oxyfluorfen & 720 & $100,0 \mathrm{a}$ & $100,0 \mathrm{a}$ & $99,7 \mathrm{a}$ & $92,5 \mathrm{a}$ \\
imazapyr & 250 & $100,0 \mathrm{a}$ & $100,0 \mathrm{a}$ & $100,0 \mathrm{a}$ & $100,0 \mathrm{a}$ \\
\hline CV $(\%)$ & 3,6 & 3,8 & 4,5 & 9,4 \\
\hline
\end{tabular}

Médias seguidas pela mesma letra, em uma coluna, não diferem entre si a 5\% de probabilidade, pelo teste de Tukey. 
O oxyfluorfen e o imazapyr são herbicidas que têm maior ação residual no solo, apresentando-se, eficiente no controle de Galinsoga parviflora, até aos 90 (dias após tratamento). O período residual do oxadiazil foi de apenas 45 dias.

A percentagem de controle total das plantas daninhas para oxadiazil, na menor dose, foi em torno a 90,5\%, até aos 45 (dias após o tratamento) (Tabela 5). Aos 90 DAT, a eficiência diminuiu para 70 e $82,3 \%$, nas doses $600 \mathrm{~g} \mathrm{ha}^{-1}$ (menor dose) e $1.000 \mathrm{~g} \mathrm{ha}^{-1}$ (maior dose), respectivamente. $\mathrm{O}$ oxadiazon propiciou um controle de 75 e $87,5 \%$ nas doses 1.140 e $1.900 \mathrm{~g} \mathrm{ha}^{-1}$, respectivamente, até os 45 DAT.

TABELA 5. Percentagem de controle total de plantas daninhas em cultura de Pinus caribea var. hondurensis, aos 45, 70 e 90 dias após a aplicação dos herbicidas.

\begin{tabular}{lcccc}
\hline \multicolumn{1}{c}{ Tratamentos } & $\begin{array}{c}\text { Doses } \\
\left(\mathrm{g} \mathrm{ha}^{-1}\right)\end{array}$ & $45 \mathrm{DAT}$ & $70 \mathrm{DAT}$ & $90 \mathrm{DAT}$ \\
\hline testemunha s/ capina & - & $0,0 \mathrm{f}$ & $0,0 \mathrm{f}$ & $0,0 \mathrm{~g}$ \\
testemunha c/ capina & - & $100,0 \mathrm{a}$ & $100,0 \mathrm{a}$ & $100,0 \mathrm{a}$ \\
oxadiazil & 600 & $90,5 \mathrm{~cd}$ & $84,5 \mathrm{bc}$ & $70,0 \mathrm{~cd}$ \\
oxadiazil & 800 & $92,3 \mathrm{bcd}$ & $90,3 \mathrm{ab}$ & $82,5 \mathrm{bc}$ \\
oxadiazil & 1000 & $95,3 \mathrm{abc}$ & $94,7 \mathrm{ab}$ & $82,5 \mathrm{cb}$ \\
oxadiazon & 1140 & $75,8 \mathrm{e}$ & $57,5 \mathrm{e}$ & $45,0 \mathrm{f}$ \\
oxadiazon & 1520 & $76,0 \mathrm{e}$ & $61,3 \mathrm{de}$ & $46,3 \mathrm{ef}$ \\
oxadiazon & 1900 & $87,5 \mathrm{~d}$ & $71,3 \mathrm{~cd}$ & $62,5 \mathrm{de}$ \\
oxyfluorfen & 720 & $96,8 \mathrm{abc}$ & $91,8 \mathrm{ab}$ & $86,3 \mathrm{abc}$ \\
imazapyr & 250 & $99,0 \mathrm{ab}$ & $98,5 \mathrm{a}$ & $95,8 \mathrm{ab}$ \\
\hline CV (\%) & & 3,8 & 7,4 & 10,2 \\
\hline Médias seguidas pela mesma a & &
\end{tabular}

Médias seguidas pela mesma letra, em uma coluna, não diferem entre si a 5\% de probabilidade, pelo teste de Tukey.

$\mathrm{O}$ oxyfluorfen em sua dose única $\left(720 \mathrm{~g} \mathrm{ha}^{-1}\right)$ propiciou controle de $96,6 \%$ das plantas daninhas, entre os 45 e os 90 DAT (Tabela 5). O imazapyr foi mais eficiente que os demais herbicidas. O controle dessas plantas daninhas variou de 99 a $95,8 \%$, entre as épocas 45 e 90 (dias após o tratamento). Segundo Gardiner \& Yeiser (1993), o imazapyr aplicado isolado ou em mistura com sulfometuron, mostrou-se seletivo para as mudas de Pinus taeda e apresentou alto controle das plantas daninhas presentes na área experimental.

Segundo Warren \& Hess (1994), oxyfluorfen pertence ao grupo de herbicidas dos difenil-ésteres e juntamente com o oxadiazon têm como mecanismo de ação inibidores de protoporfirinogênio oxidase (Protox). Quando aplicados em pré-emergência das plantas daninhas, as ações desses produtos acontecem durante a emergência das plântulas, próxima à superfície do solo, isto é, numa faixa de $0-5 \mathrm{~cm}$ ao nível do solo. Comportamento semelhante foi observado para o oxadiazil.

Oxadiazil, oxadiazon e oxyfluorfen não afetaram as plantas de pinus em nenhuma das doses estudadas. As plantas de pinus tratadas com imazapyr, na dose $250 \mathrm{~g} \mathrm{ha}^{-1}$, exibiram manchas necróticas nas acículas novas, com parcial 
recuperação do crescimento, aos 30 dias após o tratamento. Aos 90 (dias após o tratamento), as plantas apresentavam acículas novas normais, embora com crescimento menor. Isto pode limitar a aplicação sobre o dossel das mudas de pinus.

Em geral, os danos, provocados pelo imazapyr, atingiram o meristema apical, e aos 90 (dias após o tratamento), ainda foi observado crescimento abaixo do normal das plantas de pinus, embora Christoffoleti et al. (1997), tenha observado que o imazapyr é totalmente seletivo para Pinus taeda, que tolera aplicações antes e após o transplante das mudas, cujas doses, proporcionam um residual mais prolongado de controle das plantas daninhas.

O oxadiazil apresentou excelente controle de B. plantaginea, I. grandifolia e G. parviflora. Não foi eficiente para o controle de B. pilosa; entretanto proporcionou controle superior ao observado para o oxadiazon.

O oxadiazon foi eficiente até 45 (dias após tratamento) para o controle de B. plantaginea, $G$. parviflora e até 90 dias para I. grandifolia. Tanto o oxyfluorfen quanto o imazapyr apresentaram excelente controle de todas as espécies.

\section{LITERATURA CITADA}

AGUNDIS, O.M., VALTIERA, A., CASTILHO, B. Períodos críticos da competência entre frejol e malezas. Agric., México, v. 2, p. 87-90, 1962-63.

BRESDALE, J.K.A. Studies on competition. In: HARPER, J.L. The Biology of weed. Oxford: Black Weed Science, 1960. p. 133142.

CHRISTOFFOLETI, P.J., VICTORIA FILHO, R., COELHO, J.V.G., BRITVA, M. Manejo de plantas daninhas na implantação da cultura do pinus através do herbicida imazapyr. In:CONGRESSO BRASILEIRO DA CIÊNCIA DAS PLANTAS DANINHAS, 21, 1997. Resumos... Caxambu: SBCPD. p.482.
DAVIES, R.J. Arboles y malezas. Edimburgo: Asociacion Latino Americana de Malezas, 1990. s.p.

FRANS, R.W. Measuring plant response. In: WILKINSON, R.E., ed. Research methods in weed science. Puerto Rico: Weed Science Society, 1972. p.28-41.

GARDINER, E.S., YEISER, J.L. First-year survival and growth of loblolly pine seedlings released from perennial weeds in old fields of northeastern Arkansas. TreePlanters'-Notes, Monticello, v. 44, n. 1, p. 25 - 32, 1993.

PITELLI, R.A., KARAN, D. Ecologia das plantas daninhas e sua interferência em culturas florestais. In: SEMINÁRIO TÉCNICO SOBRE PANTAS DANINHAS E O USO DE HERBICIDAS EM REFLORESTAMENTO, 1, 1988. Anais... Rio de Janeiro, s.e. p. 1-20, 1988.

SILVA, W., SILVA, J.F., CARDOSO, A.A., BARROS, N.F. Utilização da trifluralina 600 e do oxyfluorfem na cultura de Eucalyptus grandis Hill ex Maiden. Rev. Árvore, v . 19, n.1, p. 1-17, 1995.

VOELLER, J.E.,YOUNG, J.F., HOLT, H.A. Seedling pine response to first-year vegetation control. Proc. Southern Weed Sci. Soc., v . 27,p. 59-63, 1974.

WARREN, G.I., HESS, F.D. Diphenylethrs and oxydiazon. In: HERBICIDE antion course. West Lafayet: Purdue University, 1994. p.128-142.

ZEN, S. Influência da matocompetição em plantio de Eucalyptus grandis. IPEF. Seminário sobre herbicidas em reflorestamento. Piracicaba, v. 4 , p. 25-35, (Série técnica). 